\title{
MODULE HOMOMORPHISMS OF A VON NEUMANN ALGEBRA INTO ITS CENTER( $\left.{ }^{1}\right)$
}

\author{
BY \\ HERBERT HALPERN
}

1. Introduction. A tool for studying von Neumann algebras is the space of $\sigma$-weakly continuous linear functionals. There are certain limitations in the applicability of this tool because the center of the algebra is somehow submerged and on the whole it seems $\sigma$-weakly continuous functionals are more suitable for studying factor algebras. In this article we propose another tool, viz. the $\sigma$-weakly continuous module homomorphisms of the von Neumann algebra $\mathscr{A}$, considered as a module over its center $\mathscr{Z}$, into $\mathscr{Z}$. First it is shown that every $\sigma$-weakly continuous linear functional can be written as the composition of a $\sigma$-weakly continuous module homomorphism into $\mathscr{Z}$ and a $\sigma$-weakly continuous linear functional on $\mathscr{Z}$. For these module homomorphisms there is obtained a specific form which generalizes the well-known form of $\sigma$-weakly continuous linear functionals ([1], [16]). Certain facts concerning decomposition theory result. Further $\mathscr{A}$ is the dual (in terms of module homomorphisms) of the space of all $\sigma$-weakly continuous module homomorphisms of $\mathscr{A}$ into $\mathscr{Z}$. As an example of the applicability of this tool a type I algebra is described as the second dual (in terms of module homomorphisms) of a module. Further applications are contained in a later paper.

It seems reasonable to conjecture from the ensuing proofs that considering the module structure might further illuminate the relation between a von Neumann algebra and an $A W^{*}$-algebra.

2. Extension of homomorphisms. Let $\mathscr{Z}$ be a commutative von Neumann algebra. An algebraic $\mathscr{Z}$-module $\mathscr{M}$ with norm $\|\cdot\|$ will be called a Banach $\mathscr{Z}$ module if (1) $\mathscr{M}$ is a Banach space, and (2) $\|C B\| \leqq\|C\|\|B\|$ for every $C \in \mathscr{Z}$ and $B \in \mathscr{M}$. A module homomorphism of $\mathscr{M}$ into $\mathscr{Z}$ will be called a functional and the set of all bounded functionals of the module $\mathscr{M}$ will be denoted by $\mathscr{M} \sim$. The space $\mathscr{M}^{\sim}$ with the operator bound is again a Banach $\mathscr{Z}$-module. If $\mathscr{A}$ is a von Neumann algebra with center $\mathscr{Z}$, then $\mathscr{A}$ is a Banach $\mathscr{Z}$-module. Whenever we talk about the module $\mathscr{A}$, we shall have this particular module in mind. A functional $\phi$ of the module $\mathscr{A}$ is said to be hermitian (resp. positive) if $\phi(A)$ is hermitian (resp. positive) in $\mathscr{Z}$ whenever $A$ is hermitian (resp. positive) in $\mathscr{A}$. A positive functional $\phi$ of the algebra (resp. module) $\mathscr{A}$ is said to be normal if lub $\phi\left(A_{n}\right)=\phi(A)$ for any monotonely increasing net $\left\{A_{n}\right\}$ of hermitian elements of $\mathscr{A}$ with least upper bound $A$.

Received by the editors January 23, 1969 and, in revised form, July 11, 1968.

${ }^{(1)}$ This work was partially supported by the National Science Foundation. 
Then the positive functional $\phi$ is a normal functional of the algebra (respectively, module) $\mathscr{A}$ if and only if $\phi$ (resp. the composition $f \cdot \phi)$ is an element of the associated space $\mathscr{A}_{*}$ of $\mathscr{A}$ (resp. for every $f$ in the associated space $\mathscr{Z}_{*}$ of $\mathscr{Z}$ ) [2, I, §4, Theorem 1]. If $E$ is an abelian projection with central support $P$ of the commutator $\mathscr{Z}^{\prime}$ of $\mathscr{Z}$, then $C P \rightarrow C E$ is an isomorphism of $\mathscr{Z} P$ onto $E \mathscr{Z}^{\prime} E$. For each $A$ in $\mathscr{Z}^{\prime}$ let $\tau_{E}(A)$ denote the inverse image of $E A E$ under this isomorphism. The map $\tau_{E}$ restricted to $\mathscr{A}$ is then easily seen to be a normal functional of the module $\mathscr{A}$.

The following theorem shows the relation between functionals of $\mathscr{A}$ and the module $\mathscr{A}$.

THEOREM 1. Let $\mathscr{A}$ be a von Neumann algebra with center $\mathscr{Z}$ and let $f$ be a normal functional on $\mathscr{A}$. Then there is a normal functional $\phi$ of the module $\mathscr{A}$ with $\phi(1)$ equal to the support $P$ of $f$ restricted to $\mathscr{Z}$ such that $f=f \cdot \phi$. The functional $\phi$ is unique in the following sense: if $\psi$ is a functional of the module $\mathscr{A}$ with $f=f \cdot \psi$, then $P \psi(A)$ $=\phi(A)$ for every $A$ in $\mathscr{A}$.

Proof. We may assume $f \neq 0$. Let $\Phi$ be the canonical representation of $\mathscr{A}$ on a Hilbert space $H$ induced by $f$ and let $x$ be a cyclic vector under $\Phi(\mathscr{A})$ in $H$ such that $f(A)=w_{x} \cdot \Phi(A)=(\Phi(A) x, x)$ for every $A$ in $\mathscr{A}[2, \mathrm{I}, \S 4]$. There is a central projection $Q$ such that $\mathscr{A}(1-Q)$ is the kernel of $\Phi$ and such that $\Phi$ carries $\mathscr{A} Q$ isomorphically onto $\Phi(\mathscr{A})$. We observe that $1-P$ is in the kernel of $\Phi$ and so $Q \leqq P$. However, $f(P-Q)=w_{x}(\Phi(P-Q))=0$; therefore $P=Q$. Now the inverse $\theta$ of $\Phi$ restricted to $\mathscr{A} P$ is a $\sigma$-weakly continuous homomorphism of $\Phi(\mathscr{A})$ onto $\mathscr{A} P[2, \mathrm{I}, \S 4$, Corollaries, Theorem 2]. Let $E$ be the abelian projection of the commutator $\Phi(\mathscr{Z})^{\prime}$ of $\Phi(\mathscr{Z})$ on $H$ corresponding to the subspace closure $\{A x \mid A \in \Phi(\mathscr{Z})\}[2, \mathrm{I}, \S 2$, no. 1, and $\S 6$, Proposition 4, Corollary 2]. Let $\tau=\tau_{E}$. Then we can easily verify that the functional $\phi=\theta \cdot \tau \cdot \Phi$ of the module $\mathscr{A}$ into $\mathscr{Z} P$ is normal, and that $f(A)=f(\Phi(A))$.

Now let $\psi$ be a functional of the module $\mathscr{A}$ such that $f \cdot \psi=f$. There is a vector $y$ in the Hilbert space of $\mathscr{A}$ such that $f=w_{y}$ on $\mathscr{Z}$ [2, III, $\S 1$, Corollary to Theorem 4]. We have that $(\psi(A) B y, C y)=(\phi(A) B y, C y)$ for every $B$ and $C$ in $\mathscr{Z}$ and every $A$ in $\mathscr{A}$. If $F$ is the projection corresponding to closure $\{B y \mid B \in \mathscr{Z}\}$, then $\psi(A) F=\phi(A) F$ for every $A$ in $\mathscr{A}$. Because the central support of $F$ is $P$, we have that $\psi(A) P=\phi(A)$ for every $A$ in $\mathscr{A}$. Q.E.D.

We now determine the form of a normal functional in terms of the trace class (cf. $[9, \S 4])$.

THEOREM 2. Let $\mathscr{A}$ be a von Neumann algebra on a Hilbert space $H$. If $\phi$ is a normal functional of the module $\mathscr{A}$, then there is a positive element $B$ in the trace class of the commutator $\mathscr{Z}^{\prime}$ of the center $\mathscr{Z}$ of $\mathscr{A}$ such that $\phi(A)=\operatorname{Tr}(A B)$.

Proof. There is a set $\left\{P_{i} \mid i \in S\right\}$ of mutually orthogonal projections of $\mathscr{Z}$ of sum 1 such that each algebra $\mathscr{Z} P_{i}$ on $P_{i} H$ has a separating unit vector $x_{i}[2, \mathrm{I}, \S 2]$. Since the functional $w_{x_{i}} \cdot \phi=f_{i}$ restricted to $\mathscr{A} P_{i}$ is normal, there is a normal functional $g_{i}$ of the commutator $\mathscr{Z}^{\prime} P_{i}$ of $\mathscr{Z} P_{i}$ on $P_{i} H$ that coincides with $f_{i}$ on $\mathscr{A} P_{i}$. However, by 
Theorem 1 there is a normal functional $\psi_{i}$ of the module $\mathscr{Z}^{\prime} P_{i}$ such that $\psi_{i}\left(P_{i}\right) \leqq P_{i}$ and $g_{i} \cdot \psi_{i}=g_{i}$. There is a $y_{i}$ in $P_{i} H$ such that $g_{i}(A)=\left(A y_{i}, y_{i}\right)$ for every $A$ in $\mathscr{Z} P_{i}$. Then $\left(A y_{i}, y_{i}\right)=f_{i}(A)=\left(A \phi(1) x_{i}, x_{i}\right)$ for every $A$ in $\mathscr{Z} P_{i}$. So $\left(\phi(1) \psi_{i}(A) B x_{i}, C x_{i}\right)$ $=\left(\phi(A) B x_{i}, C x_{i}\right)$ for every $A$ in $\mathscr{A} P_{i}$ and $B$ and $C$ in $\mathscr{Z} P_{i}$. Because $x_{i}$ is separating for $\mathscr{Z} P_{i}$ we see that $\phi(A)=\phi(1) \psi_{i}(A)$ for every $A$ in $\mathscr{A} P_{i}$. There is a unique positive element $B_{i}$ in the trace class of $\mathscr{Z}^{\prime} P_{i}$ such that $\phi(1) \psi_{i}(A)=\operatorname{Tr}\left(A B_{i}\right)$ for every $A$ in $\mathscr{Z}^{\prime} P_{i}$ [9, Theorem 4.13]. But for every finite subset $\pi$ of $S$ we have that $\operatorname{Tr}\left(\sum_{\pi} B_{i}\right)$ $\leqq \phi(1)$. So $B=\sum B_{i}$ is a positive element of the trace class of $\mathscr{Z}^{\prime}$ such that $\operatorname{Tr}(A B)$ $=\phi(A)$ for every $A$ in $\mathscr{A}$ since $\operatorname{Tr}(A B) P_{i}=\operatorname{Tr}\left(\left(A P_{i}\right) B_{i}\right)=\phi\left(A P_{i}\right)=\phi(A) P_{i}$ for every $P_{i}$. Q.E.D.

COROLlaRY. Let $\mathscr{A}$ be a von Neumann algebra with center $\mathscr{Z}$. If $\phi$ is a normal functional of the module $\mathscr{A}$, there is a monotone decreasing sequence $\left\{B_{n}\right\}$ of elements in $\mathscr{Z}^{+}$and an orthogonal sequence $\left\{E_{n}\right\}$ of abelian projections of the commutator $\mathscr{Z}^{\prime}$ of $\mathscr{Z}$ such that (1) $\lim B_{n}=0$ (uniformly); (2) $\sum B_{n}$ is bounded above; and (3) $\phi(A)=\sum B_{n} \tau_{E_{n}}(A)$ for every $A \in \mathscr{A}$.

Proof. This results from $[9, \S 2$ and $\S 4]$.

REMARK. Theorem 2 gives rise to a representation of a normal functional $f$ on a von Neumann algebra $\mathscr{A}$ as an integral over the spectrum $Z$ of the center $\mathscr{Z}$ of $\mathscr{A}$. Let $\nu$ be the so-called spectral measure on $Z$ induced by the relation $f(A)$ $=\int_{Z} A^{\wedge}(\zeta) d \nu(\zeta)(A \in \mathscr{Z})$. Here $A^{\wedge}$ denotes the Gelfand transform of $A$ in $\mathscr{Z}$. There is a positive element $B$ in the trace class of the commutator of $\mathscr{Z}$ such that $f(A)=f(\operatorname{Tr}(A B))=\int \operatorname{Tr}(A B)^{\wedge}(\zeta) d \nu(\zeta)$. In the same notation as the preceding corollary let $f_{n \zeta}(A)=\left(B_{n} \tau_{E_{n}}(A)\right)^{\wedge}(\zeta)$ for every $\zeta \in Z$. Then $f_{n \zeta}$ is a positive functional on $\mathscr{A}$ whose kernel contains the ideal $\zeta$ in $Z$. If $\mathscr{A}$ is discrete, the nonzero $f_{n \zeta}$ are irreducible (cf. [8, Proposition 2.1]). If $\mathscr{A}$ is continuous, then $f_{n \zeta}(1)^{-1} f_{n \zeta}$ is in the pure state space of $\mathscr{A}$ whenever $f_{n \zeta} \neq 0$ [4, Theorem 3]. Now for fixed $A \in \mathscr{A}^{+}$ $\left\{\sum\left\{B_{n} \tau_{E_{n}}(A) \mid 1 \leqq n \leqq m\right\}\right\}_{m}$ is a monotonely increasing sequence which converges strongly to $\operatorname{Tr}(A B)$. There is a subset $N$ of $\nu$-measure 0 such that

$$
\lim _{m} \sum\left\{f_{n \zeta}(A) \mid 1 \leqq n \leqq m\right\}=\operatorname{Tr}(B A)^{\wedge}(\zeta)
$$

for every $\zeta \notin N$. The restriction that $A \in \mathscr{A}^{+}$can certainly be removed and so, we obtain the formula $\sum_{n} \int f_{n \zeta}(A) d \nu(\zeta)=\int \operatorname{Tr}(B A)^{\wedge}(\zeta) d \nu(\zeta)=f(A)$ for every $A$ in $\mathscr{A}$ by the dominated convergence theorem.

We state some additional facts for type I algebras. Here we may assume $\mathscr{A}=\mathscr{Z}^{\prime}$. The support $X$ of $\nu$ is open and closed and corresponds to a projection $P$ in $\mathscr{Z}$ under the relation $\left\{\zeta \in Z \mid P^{\wedge}(\zeta)=1\right\}=X$. Let $E$ be an abelian projection of central support $P$. If $\zeta \in X$ and if [ $\zeta]$ denotes the smallest closed two-sided ideal in $\mathscr{A}$ containing $\zeta$, then the factor space $\mathscr{A} E /[\zeta]$ becomes a Hilbert space $H(\zeta)$ with inner product $(A(\zeta), B(\zeta))=\tau_{E}\left(B^{*} A\right)^{\wedge}(\zeta)$. Here $A(\zeta)$ denotes the image of $A$ in $\mathscr{A} /[\zeta]$. Let $\Phi_{\zeta}$ be the

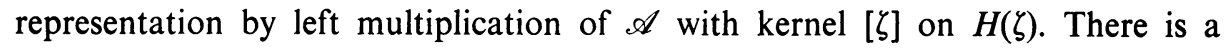


sequence $\left\{P_{k}\right\}$ of mutually orthogonal projections in $\mathscr{Z}$ of sum $P$ such that $\left\{\sum_{n} B_{n} P_{k}\right\}$ converges uniformly for each $P_{k}$ (cf. [9, Theorem 4.1]). Let $X_{k}=\left\{\zeta \in Z \mid P_{k}(\zeta)=1\right\}$. For each $\zeta \in \bigcup X_{k}$ there is a normal functional $f_{\zeta}$ on the algebra $L$ of bounded linear operators of $H(\zeta)$ with $f_{\zeta} \cdot \Phi_{\zeta}(A)=\operatorname{Tr}(B A)^{\wedge}(\zeta)$. Indeed, there is a normal functional $g_{n \xi}$ on $L$ with $g_{n \xi} \cdot \Phi_{\xi}=f_{n \xi}$. Since the set $N=X-\bigcup X_{k}$ has $\nu$-measure 0 , we have that $f(A)=\int f_{\zeta}(A(\zeta)) d \nu(\zeta),[9, \S 4]$.

Let $\mathscr{A}$ be a von Neumann algebra with center $\mathscr{Z}$. The set $\mathscr{A}_{\sim}$ of all functionals of the module $\mathscr{A}$ which are continuous in the $\sigma\left(\mathscr{A}, \mathscr{A}_{*}\right)$ and $\sigma\left(\mathscr{Z}, \mathscr{Z}_{*}\right)$ topologies of $\mathscr{A}$ and $\mathscr{Z}$ respectively is an algebraic $\mathscr{Z}$-module. The module $\mathscr{A}_{\sim}$ is a closed subspace of the space $\mathscr{A} \sim$ of all bounded functionals of the module $\mathscr{A}$ because the uniform limit of a sequence of $\sigma$-weakly continuous functionals of $\mathscr{A}$ is $\sigma$-weakly continuous. Thus $\mathscr{A}_{\sim}$ is a Banach $\mathscr{Z}$-module.

In passing we remark that analogues of theorems for functionals of $\mathscr{A}_{*}$ may be formulated and proved for functionals of $\mathscr{A}_{\sim}$ using Theorem 1. In particular, we may obtain a polar decomposition for functionals of $\mathscr{A}_{\sim}$ [14] as well as a decomposition of functionals of $\mathscr{A}_{\mathcal{L}}$ into a linear combination of normal functionals. The proof of the latter statement is virtually identical with that of Theorem 4.12 of [9]. In particular, if $\phi$ is a hermitian functional of $\mathscr{A}_{\sim}$, then there is a projection $E \in \mathscr{A}$ such that $\phi_{1}(A)=\phi(A E)$ and $\phi_{2}(A)=-\phi(A(1-E))$ are normal functionals of the module $\mathscr{A}$. Also a Radon-Nikodym theorem [15] holds.

The next theorem is an analogue of a well-known result concerning linear functionals on a von Neumann algebra. The theorem though is potentially applicable to the theory of $A W^{*}$-algebras in a way precluded for linear functionals (cf. [13]) and so is interesting in its own right. We plan to explore this application in a future paper.

THEOREM 3. Let $\mathscr{A}$ be a von Neumann algebra with center $\mathscr{Z}$. For each $A$ in $\mathscr{A}$ define the function $F_{A}$ on $\mathscr{A}_{\sim}$ by $F_{A}(\phi)=\phi(A)$. Then the function $A \rightarrow F_{A}$ defines an isometric isomorphism of the module $\mathscr{A}$ onto the module $\left(\mathscr{A}_{\sim}\right)^{\sim}$.

Proof. First it is easy to see that $F_{A} \in\left(\mathscr{A}_{\sim}\right)^{\sim}$ for each $A \in \mathscr{A}$ and that the function $A \rightarrow F_{A}$ is a module homomorphism of $\mathscr{A}$ into $\left(\mathscr{A}_{\sim}\right)^{\sim}$. Now let $\mathscr{Z}^{\prime}$ be the commutator of $\mathscr{Z}$; let $\varepsilon>0$ be given. There is an abelian projection $E$ in $\mathscr{Z}^{\prime}$ such that $\|E|A| E\| \geqq\|A\|-\varepsilon$ where $|A|=\left(A^{*} A\right)^{1 / 2}$. Let $U$ be a partial isometry of $\mathscr{A}$ such that $A=U|A|$ is the polar decomposition of $A$. Defining $\phi(B)=\tau_{E}\left(U^{*} B\right)$ for $B \in \mathscr{A}$, we see that $\|\phi(B)\|^{2} \leqq\left\|\tau_{E}\left(U^{*} U\right)\right\|\left\|\tau_{E}\left(B^{*} B\right)\right\| \leqq\|B\|^{2}$ for every $B \in \mathscr{A}$. Thus $\phi$ is in the unit sphere of $\mathscr{A}_{\sim}$. However $\left\|F_{A}(\phi)\right\| \geqq\|A\|-\varepsilon$. Because $\varepsilon>0$ is arbitrary $\left\|F_{A}\right\| \geqq\|A\|$. Since it is obviously true that $\left\|F_{A}\right\| \leqq\|A\|$, we have that $\left\|F_{A}\right\|=\|A\|$. Hence the map $A \rightarrow F_{A}$ is an isometry.

We complete the proof by showing $A \rightarrow F_{A}$ is onto $\left(\mathscr{A}_{\sim}\right)^{\sim}$. Let $f$ be an element of $\left(\mathscr{A}_{\sim}\right)^{\sim}$. For each $\phi$ in $\mathscr{Z}_{\sim}^{\prime}$ let $F(\phi)$ denote the restriction of $\phi$ to $\mathscr{A}$. Then $F$ is a continuous homomorphism of the $\mathscr{Z}$-module $\mathscr{Z}_{\sim}^{\prime}$ onto the $\mathscr{Z}$-module $\mathscr{A}_{\sim}$. This means that $f \cdot F$ is an element of $\left(\mathscr{Z}_{\sim}^{\prime}\right)^{\sim}$. There is an element $A$ in $\mathscr{Z}^{\prime}$ such that 
$f \cdot F(\phi)=\phi(A)$ for every $\phi$ in $\mathscr{Z}_{\sim}^{\prime}$ [9, Theorems 4.10 and 4.13]. We show that $A \in \mathscr{A}$. Indeed, if $A \notin \mathscr{A}$ then there is a unitary operator $V$ in the commutator $\mathscr{A}^{\prime}$ of $\mathscr{A}$ such that $V A V^{*} \neq A$. There is an abelian projection $G$ in $\mathscr{Z}^{\prime}$ such that $G V A V^{*} G$ $\neq G A G$. Let $G^{\prime}$ be the abelian projection in $\mathscr{Z}^{\prime}$ given by $G^{\prime}=V^{*} G V$. If $\phi=\tau_{G^{\prime}}-\tau_{G}$, then $\phi(B)=0$ for every $B$ in $\mathscr{A}$; but $\phi(A)=\tau_{G}\left(V A V^{*}-A\right) \neq 0$. Thus $0=f \cdot F(\phi)$ $=\phi(A) \neq 0$. This is impossible. So we conclude that $A \in \mathscr{A}$. Now by the preliminary remarks concerning the decomposition of elements of $\mathscr{A}_{\sim}$ into a linear combination of normal functionals and by Theorem 2 we find that the range of $F$ is $\mathscr{A}_{\sim}$. If $\phi \in \mathscr{A}_{\sim}$, then there is a $\psi \in \mathscr{Z}_{\sim}^{\prime}$ such that $F(\psi)=\phi$ and thus $f(\phi)=\psi(A)=\phi(A)$ since $A \in \mathscr{A}$. This completes the proof. Q.E.D.

3. Characterization of type I algebras. It is known that a $C^{*}$-algebra that can be expressed as the second dual of a Banach space is a von Neumann algebra with minimal projections ([13], [7], [19]). So linear functionals are suitable for characterizing type I factor algebras but unsuitable for characterizing algebras with arbitrary centers. The object of this section is to bring the center into consideration by considering module homomorphisms and thereby to obtain a description of type I algebras.

THEOREM 4. Let $\mathscr{Z}$ be a commutative von Neumann algebra and let $\mathscr{A}$ be a $C^{*}$-algebra with center $\mathscr{Z}$. Let $\mathscr{M}$ be a Banach $\mathscr{Z}$-module. If $\mathscr{A}$ is the dual $\mathscr{M} \sim$ of $\mathscr{M}$, then $\mathscr{A}$ is a von Neumann algebra and every functional $A \rightarrow \phi(A)$ of $\mathscr{A}$ into $\mathscr{Z}$ for fixed $\phi$ in $\mathscr{M}$ is a $\sigma$-weakly continuous functional of the module $\mathscr{A}$.

Note. We write $\phi(A)$ to indicate the functional $A \in \mathscr{A}$ applied to $\phi \in \mathscr{M}$.

Proof. Let $\mathscr{B}$ be the linear manifold in the dual $\mathscr{A}^{*}$ of $\mathscr{A}$ generated by the set $\left\{f \cdot \phi \mid f \in \mathscr{Z}_{*}, \phi \in \mathscr{M}\right\}$. We show that the dual $\mathscr{B}^{*}$ of $\mathscr{B}$ is identified with $\mathscr{A}$. Then by Sakai's Theorem [13, Theorem 1 and Remark 1] $\mathscr{A}$ is a von Neumann algebra and the functionals of $\mathscr{B}$ are $\sigma$-weakly continuous on $\mathscr{A}$. In fact the closure of $\mathscr{B}$ in $\mathscr{A}^{*}$ is equal to the associated space of $\mathscr{A}[12,22.6]$. This means that the elements of $\mathscr{M}$ are $\sigma$-weakly continuous. We proceed to identify $\mathscr{B}^{*}$ with $\mathscr{A}$. Let $\Phi$ be an element of $\mathscr{B}^{*}$. Let $H$ be a Hilbert space on which $\mathscr{Z}$ may be represented as a von Neumann algebra. The functional $w_{x, y} \cdot \phi$ is in $\mathscr{B}$ for every $x$ and $y$ in $H$ and $\phi \in \mathscr{M}$. Here $w_{x, y}(A)=(A x, y)$. Setting $\langle x, y\rangle_{\phi}=\Phi\left(w_{x, y} \cdot \phi\right)$ we obtain for each $\phi \in \mathscr{M}$ a bilinear form on $H$. Since $\left|\langle x, y\rangle_{\phi}\right| \leqq\|x\|\|y\|\|\phi\|\|\Phi\|$, the form is continuous and hence there is a unique bounded linear operator $A_{\phi}$ on $H$ such that $\langle x, y\rangle_{\phi}=\left(A_{\phi} x, y\right)$ for every $x$ and $y$ in $H$. If $B$ is an arbitrary element in the commutator $\mathscr{Z}^{\prime}$ of $\mathscr{Z}$ on $H$, then $\left(A_{\phi} B x, y\right)=\left(B A_{\phi} x, y\right)$ for every $x$ and $y$ in $H$ because $w_{B x, y} \cdot \phi=w_{x, B^{*} y} \cdot \phi$. This means $A_{\phi}$ is in the double commutator of $\mathscr{Z}$ which is $\mathscr{Z}$. The map $\phi \rightarrow A_{\phi}$ is easily seen to be a module homomorphism of $\mathscr{M}$ into $\mathscr{Z}$. It is bounded because

$$
\left\|A_{\phi}\right\|=\operatorname{lub}\left\{\left|\Phi\left(w_{x, y} \cdot \phi\right)\right| \mid\|x\| \leqq 1,\|y\| \leqq 1\right\} \leqq\|\Phi\|\|\phi\| .
$$

By hypothesis there is a unique $A_{0}$ in $\mathscr{A}$ such that $\phi\left(A_{0}\right)=A_{\phi}$ for every $\phi \in \mathscr{M}$. Then for every $g \in \mathscr{B}$ we have that $\Phi(g)=g\left(A_{0}\right)$. 
For each $A \in \mathscr{A}$ let $G_{A}$ be the element of $\mathscr{B} *$ defined by $G_{A}(g)=g(A)$. The map $A \rightarrow G_{A}$ of $\mathscr{A}$ into $\mathscr{B}^{*}$ is a linear function of $\mathscr{A}$ whose range is $\mathscr{B}^{*}$ by the preceding paragraph. We complete the proof by showing that this map is an isometry. Because $\mathscr{B}$ is a subset of $\mathscr{A}^{*}$ we have that $\left\|G_{A}\right\| \leqq\|A\|$. On the other hand the two relations

$$
\operatorname{lub}\{\|\phi(A)\| \mid \phi \in \mathscr{M},\|\phi\| \leqq 1\}=\|A\|
$$

and

$$
\operatorname{lub}\left\{|f \cdot \phi(A)| \mid f \in \mathscr{Z}_{*},\|f\| \leqq 1\right\}=\|\phi(A)\|
$$

imply the relation $\|A\| \leqq\left\|G_{A}\right\|$. So $\|A\|=\left\|G_{A}\right\|$. Q.E.D.

Before proceeding we make some observations. Assume that $\mathscr{M}$ is a Banach modulo over a commutative von Neumann algebra $\mathscr{Z}$ whose second dual $\mathscr{M}^{\sim}$ is a $C^{*}$-algebra $\mathscr{A}$ with center $\mathscr{Z}$. Let $\mathscr{M}^{\prime}$ be the Banach $\mathscr{Z}$-module $\mathscr{M}$ reduced module $\mathscr{K}=\bigcap\left\{\right.$ kernel $\left.\phi \mid \phi \in \mathscr{M}^{\sim}\right\}$ with the norm $\left\|A^{\prime}\right\|=\operatorname{glb}\{\|A+B\| \mid B \in \mathscr{K}\}$. Here $A^{\prime}$ is the image in $\mathscr{M}^{\prime}$ of $A$ in $\mathscr{M}$ under the canonical homomorphism. It is standard to show that $\mathscr{M}^{\sim}$ is identified with (i.e. is isometric isomorphic to) the dual $\mathscr{M}^{\prime} \sim$ under the correspondence $\phi \rightarrow \phi^{\prime}$ where $\phi^{\prime}\left(A^{\prime}\right)=\phi(A)$. By replacing $\mathscr{M}$ by $\mathscr{M}^{\prime}$ if necessary we may assume that $\mathscr{K}=(0)$. For each $A \in \mathscr{M}$ the function $\phi \rightarrow \phi(A)$ defines an element $F_{A}$ of $\mathscr{M}^{\sim \sim}=\mathscr{A}$. The map $A \rightarrow F_{A}$ is an isomorphism of $\mathscr{M}$ into a submodule $\mathscr{N}$ of the module $\mathscr{A}$. Setting $\phi^{\prime}(A)=\phi\left(F_{A}\right)$ for each $\phi \in \mathscr{N} \sim$ and $A \in \mathscr{M}$, we obtain a functional $\phi^{\prime}$ of $\mathscr{M}^{\sim}$ because $\left\|F_{A}\right\| \leqq\|A\|$. It satisfies the relation

$$
\begin{aligned}
\|\phi\| & =\operatorname{lub}\{\|\phi(A)\| \mid A \in \mathscr{N},\|A\| \leqq 1\} \\
& \geqq \operatorname{lub}\left\{\left\|\phi^{\prime}(A)\right\| \mid A \in \mathscr{M},\|A\| \leqq 1\right\}=\left\|\phi^{\prime}\right\| \\
& \geqq \operatorname{lub}\left\{\left\|\phi^{\prime}(A)\right\| \mid A \in \mathscr{A},\|A\| \leqq 1\right\} \geqq\|\phi\| .
\end{aligned}
$$

This means that $\phi \rightarrow \phi^{\prime}$ is an isometric isomorphism of $\mathscr{N} \sim$ onto $\mathscr{M} \sim$. Because it is well known that the dual of the closure of $\mathscr{N}$ is equal to $\mathscr{N} \sim$, we may assume that $\mathscr{M}$ is embedded in $\mathscr{A}$. For every $A$ in $\mathscr{M}$ we have that

$$
\|A\|=\operatorname{lub}\left\{\|\phi(A)\| \mid \phi \in \mathscr{M}^{\sim},\|\phi\| \leqq 1\right\}
$$

because $\mathscr{M}^{\sim \sim}=\mathscr{A}$ and for every $\phi \in \mathscr{M}^{\sim}$ we have that

$$
\begin{aligned}
\|\phi\| & =\operatorname{lub}\{\|\phi(A)\| \mid A \in \mathscr{M},\|A\| \leqq 1\} \\
& \leqq \operatorname{lub}\{\|\phi(A)\| \mid A \in \mathscr{A},\|A\| \leqq 1\} \leqq\|\phi\| .
\end{aligned}
$$

So we are always justified in assuming that $\mathscr{M}$ is embedded in $\mathscr{A}$ and that (1) and (2) hold.

TheOREM 5. Let $\mathscr{A}$ be a von Neumann algebra with center $\mathscr{Z}$. If $\mathscr{M}$ is a closed submodule of the module $\mathscr{A}$ whose second dual $\mathscr{M} \sim \sim$ is equal to $\mathscr{A}$, then $\mathscr{M}$ is $\sigma$-weakly dense in $\mathscr{A}$. 
Proof. We use the same notation as Theorem 4. Every $f \in \mathscr{B}$ has the form $f=\sum\left\{f_{i} \cdot \phi_{i} \mid 1 \leqq i \leqq n\right\}$ where $f_{i} \in \mathscr{Z}_{*}$ and $\phi_{i} \in \mathscr{M}^{\sim}$. Because every $f_{i}$ may be written as a linear combination of positive functionals in $\mathscr{Z}_{*}$, we may assume every $f_{i}$ is positive. Then $\sum f_{i}=w_{x}$ for some $x \in H$. By the Radon-Nikodym theorem there is a $C_{i} \in \mathscr{Z}^{+}$such that $f_{i}=w_{C_{i} x}(1 \leqq i \leqq n)$. Hence we may assume that $f$ has the form $f=w_{x} \cdot \phi$ for some $\phi \in \mathscr{M}^{\sim}$.

We now obtain a contradiction by assuming that the complement of the weak closure $\mathscr{N}$ of the unit sphere of $\mathscr{M}$ with respect to the unit sphere $\mathscr{A}_{1}$ of $\mathscr{A}$ contains an element $B$. Standard separation theorems $[12,14.2]$ give an $f \in \mathscr{A}_{*}$ such that lub $\{\operatorname{Re} f(A) \mid A \in \mathscr{N}\}<\operatorname{Re} f(B)$. Here $\operatorname{Re} \alpha$ denotes the real part of $\alpha$. Because $\mathscr{B}$ is uniformly dense in $\mathscr{A}_{*}$ and because $\mathscr{N} \subset \mathscr{A}_{1}$, there is by the preceding paragraph an $x \in H$ and a $\phi \in \mathscr{M}^{\sim}$ such that lub $\left\{\operatorname{Re} w_{x} \cdot \phi(A) \mid A \in \mathscr{N}^{\prime}\right\}<\operatorname{Re} w_{x} \cdot \phi(B)$. We show that $\{\operatorname{Re} \phi(A) \mid A \in \mathscr{N}\}=X$ is monotonely increasing in $\mathscr{Z}$. Here $\operatorname{Re} \phi(A)=$ $\left(\phi(A)+\phi(A)^{*}\right) / 2$. For $A_{i}(i=1,2)$ in $\mathscr{N}$ let $U_{i}^{*}\left|\phi\left(A_{i}\right)\right|=\phi\left(A_{i}\right)$ be the polar decomposition of $\phi\left(A_{i}\right)$ in $\mathscr{Z}$. The element $\phi\left(U_{i} A_{i}\right)$ is in $X$ because $U_{i} A_{i} \in \mathscr{N}$. Note that $\operatorname{Re} \phi\left(A_{i}\right) \leqq\left|\phi\left(A_{i}\right)\right|=\phi\left(U_{i} A_{i}\right)$. There is a central projection $P$ such that $P \phi\left(U_{1} A_{1}\right)$ $\leqq P \phi\left(U_{2} A_{2}\right)$ and $(1-P) \phi\left(U_{2} A_{2}\right) \leqq(1-P) \phi\left(U_{1} A_{1}\right)$ and so the element $A=P U_{2} A_{2}$ $+(1-P) U_{1} A_{1}$ in $\mathscr{N}$ satisfies the relation $\operatorname{Re} \phi\left(A_{i}\right) \leqq \phi(A)$ for $i=1,2$. This shows $X$ is monotonely increasing. Because $X$ is bounded above in $\mathscr{Z}$, it is possible to find a net $\left\{A_{n}\right\}$ in $\mathscr{N}$ such that $\left\{\operatorname{Re} \phi\left(A_{n}\right)\right\}$ converges strongly to the least upper bound $C_{0}$ of $X$. Because $\mathscr{N}$ is compact in the weak topology, there is a subnet $\left\{A_{n_{y}}\right\}$ which converges weakly to an element $A_{0} \in \mathscr{N}$. However $\operatorname{Re} \phi\left(A_{0}\right)=C_{0}$ due to the weak continuity of $\phi$ (Theorem 4). By multiplying $\phi\left(A_{0}\right)$ by an appropriate partial isometry in $\mathscr{Z}$ we may assume that $\phi\left(A_{0}\right)=C_{0}$. For every projection $P$ in $\mathscr{Z}$ we have $\|P \phi\|=\left\|P C_{0}\right\|$. On the contrary, if $\left\|P C_{0}\right\|<\|P \phi\|$, then $Q\left(C_{0}+\varepsilon\right) \leqq\left(\left\|P C_{0}\right\|+\varepsilon\right) Q$ $\leqq Q \phi(A)$ for suitable $A \in \mathscr{M} \cap \mathscr{A}_{1}, \varepsilon>0$ and central projection $Q$ with $0<Q \leqq P$ because $\|P \phi\|=\operatorname{lub}\left\{\|P \phi(C)\| \mid C \in \mathscr{M} \cap \mathscr{A}_{1}\right\}$. This contradicts the definition of $C_{0}$. Therefore $\|P \phi\|=\left\|P C_{0}\right\|$ for every central projection $P$. Now if $U^{*}|\phi(B)|=\phi(B)$ is the polar decomposition of $\phi(B)$, then $\operatorname{Re} w_{x}(\phi(B))=w_{x}(\operatorname{Re} \phi(B)) \leqq w_{x}(|\phi(B)|)$ $=w_{x}(\phi(U B))$. There is no loss of generality in assuming $\phi(B) \in \mathscr{Z}^{+}$. We see that $\phi(B) \leqq C_{0}$; otherwise there is a projection $P \neq 0$ in $\mathscr{Z}$ and an $\varepsilon>0$ such that $P\left(C_{0}+\varepsilon\right)$ $\leqq P \phi(B)$. This means that $\left\|P C_{0}\right\|<\|P \phi\|$ and that is impossible. However $w_{x} \cdot \phi(B)$ $\leqq w_{x}\left(C_{0}\right)=w_{x}\left(\phi\left(A_{0}\right)\right)$ is obtained. Because $A_{0} \in \mathscr{N}$ we have reached a contradiction. Therefore $\mathscr{N}=\mathscr{A}_{1}$. Q.E.D.

COROLlaRy. The module $\mathscr{M}^{\sim}$ is equal to the module $\mathscr{A}_{\sim}$ of all $\sigma$-weakly continuous functionals of the module $\mathscr{A}$.

Proof. If $\phi \in \mathscr{A}_{\sim}$, then the restriction $\phi \mid \mathscr{M}$ of $\phi$ to $\mathscr{M}$ defines a bounded functional of the module $\mathscr{M}$. There is a $\psi \in \mathscr{M}^{\sim}$ such that $\psi=\phi \mid \mathscr{M}$. Because $\psi \in \mathscr{A}_{\sim}$ (Theorem 4) and because $\mathscr{M}$ is $\sigma$-weakly dense in $\mathscr{A}$, we have that $\psi=\phi$. So $\mathscr{A}_{\sim} \subset \mathscr{M}^{\sim}$. Since $\mathscr{M}^{\sim \subset \mathscr{A}_{\sim}}$ (Theorem 4), we have $\mathscr{A}_{\sim}=\mathscr{M}^{\sim}$. Q.E.D.

Let $\mathscr{M}$ be a Banach module over the commutative von Neumann algebra $\mathscr{Z}$; 
the topology induced on the dual $\mathscr{M}^{\sim}$ of the module $\mathscr{M}$ by the family of seminorms $p_{f, A}(\phi)=|f(\phi(A))|\left(f \in \mathscr{Z}_{*}, A \in \mathscr{M}\right)$ will be called the weak topology of $\mathscr{M}^{\sim}$.

TheOREM 6. Let $\mathscr{M}$ be a Banach module over the commutative $\sigma$-finite von Neumann algebra $\mathscr{Z}$. Then the unit sphere $\mathscr{M}_{1}^{\tilde{1}}$ of $\mathscr{M}^{\sim}$ is compact in the weak topology.

Proof. Let $x$ be a separating unit vector for $\mathscr{Z}$. Let $\left\{\phi_{n}\right\}$ be a net in $\mathscr{M}_{\tilde{1}}$. A subnet $\left\{w_{x} \cdot \phi_{n} \mid n \in S\right\}$ of $\left\{w_{x} \cdot \phi_{n}\right\}$ converges in the $\sigma\left(\mathscr{M}^{*}, \mathscr{M}\right)$-topology of the dual $\mathscr{M}^{*}$ of $\mathscr{M}$ to the functional $f$ in the unit sphere of $\mathscr{M}^{*}$. Because $\left|f\left(C D^{*} B\right)\right| \leqq\|B\|\|C x\|\|D x\|$ for each $B \in \mathscr{M}$ and $C, D \in \mathscr{Z}$, the relation $\langle C x, D x\rangle_{B}=f\left(C D^{*} B\right)$ defines a continuous bilinear functional on a dense linear manifold of the subspace

$$
K=\text { closure }\{C x \mid C \in \mathscr{Z}\} .
$$

This bilinear functional may be uniquely extended to a bilinear functional $\langle y, z\rangle_{B}$ of $K$ such that $\left|\langle y, z\rangle_{B}\right| \leqq\|B\|\|y\|\|z\|$ for every $y$ and $z$. There is a unique bounded linear operator $A^{\prime}=A_{B}^{\prime}$ on $K$ such that $\left(A^{\prime} y, z\right)=\langle y, z\rangle_{B}$ for all $y$ and $z$. The projection $E$ of the Hilbert space onto $K$ is an abelian projection of the commutator $\mathscr{Z}^{\prime}$ of $\mathscr{Z}$. If $A \in \mathscr{Z}$, then $\left(A^{\prime} A E C x, D x\right)=\left(A E A^{\prime} C x, D x\right)$ for every $C$ and $D$ in $\mathscr{Z}$. Thus $(A E) A^{\prime}=A^{\prime}(A E)$, and because $A \in \mathscr{Z}$ is arbitrary, we find that $A^{\prime}$ is an element of the commutator $E \mathscr{Z}^{\prime} E$ of $\mathscr{Z} E$ on $K$. There is unique $\phi(B)$ in $\mathscr{Z}$, because the central support of $E$ is 1 , such that $\phi(B) E=A_{B}^{\prime}$. By the uniqueness of $\phi(B)$ we may conclude that $B \rightarrow \phi(B)$ is a module homomorphism of $\mathscr{M}$ into $\mathscr{Z}$ and by the relation $|(\phi(B) y, z)| \leqq\|B\|\|y\|\|z\|$ for all $y$ and $z$ in $K$ we may conclude that $\|\phi(B)\|=\|\phi(B) E\| \leqq\|B\|$. So $\phi \in \mathscr{M}_{\tilde{1}}$. From the fact that $\left\{\left\|\phi_{n}(B)\right\| \mid n \in S\right\}$ is bounded for fixed $B \in \mathscr{M}$ and from the fact that $\{C x \mid C \in \mathscr{Z}\}$ is dense in $K$, we see that $\left\{\phi_{n}(B) E \mid n \in S\right\}$ converges weakly to $\phi(B) E$. This implies that $\left\{\phi_{n}(B) \mid n \in S\right\}$ converges weakly to $\phi(B)$ for every $B \in \mathscr{M}[2, \mathrm{I}, \S 4$, Theorem 2]. Q.E.D.

A subset $\mathscr{K}$ of a module $\mathscr{M}$ over the commutative von Neumann algebra $\mathscr{Z}$ is said to be $\mathscr{Z}$-convex if $C A+(1-C) B$ is in $\mathscr{K}$ for every $A$ and $B$ in $\mathscr{K}$ and $C$ in $\mathscr{Z}$ with $0 \leqq C \leqq 1$. A nonvoid $\mathscr{Z}$-convex subset $\mathscr{K}^{\prime}$ of a $\mathscr{Z}$-convex subset $\mathscr{K}$ of $\mathscr{M}$ is said to be a support of $\mathscr{K}$ if $C A+(1-C) B \in \mathscr{K}^{\prime}$ for $A$ and $B$ in $\mathscr{K}$ and $C$ in $\mathscr{Z}$ with $0<C<1$ implies $A \in \mathscr{K}^{\prime}$ and $B \in \mathscr{K}^{\prime}$. By $0<C<1$ it is meant that $C$ is strictly between 0 and 1 , i.e. the spectrum of $C$ is contained in the open interval $(0,1)$. A point $A$ of a $\mathscr{Z}$-convex subset $\mathscr{K}$ of $\mathscr{M}$ is said to be an extreme point of $\mathscr{K}$ if $\{A\}$ is a support of $\mathscr{K}$.

The following is a Krein-Milman Theorem for $\mathscr{M}^{\sim}$.

Theorem 7. Let $\mathscr{M}$ be a Banach $\mathscr{Z}$-module whose second dual $\mathscr{M} \sim \sim$ is a von Neumann algebra $\mathscr{A}$ with center $\mathscr{Z}$. Let $P$ be a nonzero projection in $\mathscr{Z}$. Then there is an extreme point $\phi$ in the unit sphere $\mathscr{S}$ of positive functionals of $\mathscr{A}_{\sim}$ such that $P \phi \neq 0$.

Proof. We may assume that $P$ is $\sigma$-finite since every nonzero projection in $\mathscr{Z}$ majorizes a nonzero $\sigma$-finite projection. Let $\mathscr{H}=\left\{\operatorname{Re} \phi \mid \phi \in \mathscr{A}_{\sim}\right.$ and $\left.P \phi=\phi\right\}$. Here 
$\operatorname{Re} \phi(A)=\left(\phi(A)+\phi(A)^{*}\right) / 2$. The set $\mathscr{H}$ has a natural module structure over the ring of hermitian elements in $\mathscr{Z}$. The seminorms $p_{f, A}\left(f \in \mathscr{Z}_{*}, A \in \mathscr{M}\right)$ induce a weak topology on $H$ in which the $\mathscr{Z}$-convex subset $\mathscr{H}_{1}=\left\{\operatorname{Re} \phi \mid \phi \in \mathscr{A}_{\sim}, P \phi=\phi\right.$, $\|\phi\| \leqq 1\}$ is compact since the unit sphere of $\mathscr{M}^{\sim}$ is compact in the weak topology and since the adjoint operation of $\mathscr{Z}$ is weakly continuous. Suppose that $\mathscr{H}_{1}$ has a nonzero extreme point $\phi^{\prime}$. We show that $\mathscr{S}$ has a nonzero extreme point $\psi$ such that $P \psi=\psi$. Let $\phi$ be an element of the unit sphere of $\mathscr{A}_{\sim}$ such that $P \phi=\phi$ and $\operatorname{Re} \phi=\phi^{\prime}$. If $C \theta+(1-C) \theta^{\prime}=\phi$ for $\theta, \theta^{\prime}$ in the unit sphere of $\mathscr{A}_{\sim}$ and $C$ strictly between 0 and 1 in $\mathscr{Z}$, then $C \operatorname{Re} \theta+(1-C) \operatorname{Re} \theta^{\prime}=\phi^{\prime}$ and consequently $\phi^{\prime}$ $=\operatorname{Re} \theta=\operatorname{Re} \theta^{\prime}$. This means that $\phi=\theta=\theta^{\prime}$ and thus that $\phi$ is an extreme point of the unit sphere of $\mathscr{A}_{\sim}$. Using the polar decomposition for $\phi$, we obtain a partial isometric operator $U$ in $\mathscr{A} P$ such that $\psi(A)=\phi(U A)$ defines a functional in $\mathscr{S}$ and such that $\psi\left(U^{*} A\right)=\phi(A)$ for every $A$ in $\mathscr{A}$. If $\psi=C \theta+(1-C) \theta^{\prime}$ for $\theta, \theta^{\prime} \in \mathscr{S}$ and $C \in \mathscr{Z}$ with $0<C<1$, then $\phi(A)=\theta\left(U^{*} A\right)=\theta^{\prime}\left(U^{*} A\right)$ for every $A \in \mathscr{A}$. But $\psi\left(U^{*} U\right)=\psi(1)$ and so both $\theta$ and $\theta^{\prime}$ vanish on the projection $1-U^{*} U$. By the Cauchy-Schwarz inequality we have that $\theta(A)=\theta\left(U^{*} U A\right)$ and $\theta^{\prime}(A)=\theta^{\prime}\left(U^{*} U A\right)$ for every $A \in \mathscr{A}$. We then see that $\psi=\theta=\theta^{\prime}$ and thus that $\psi$ is a nonzero extreme point of $\mathscr{S}$ such that $P \psi=\psi$. We complete the proof by showing that $\mathscr{H}_{1}$ has a nonzero extreme point.

Now let $\mathscr{F}$ be a maximal family with the finite intersection property of compact supports of $\mathscr{H}_{1}$. The set $\mathscr{H}_{0}=\bigcap \mathscr{F}$ is nonvoid and evidently a compact support of $\mathscr{H}_{1}$. We show that $\mathscr{H}_{0}$ contains a single point. On the contrary if $\phi$ and $\psi$ are two distinct points in $\mathscr{H}_{0}$, there is an element $A$ in $\mathscr{M}$ such that $\phi(A) \neq \psi(A)$. Indeed, an element in $\mathscr{A}_{\sim}$ which vanishes under multiplication by $1-P$ is uniquely determined by its values on the elements of $\mathscr{M}$. The set $\left\{\theta(A) \mid \theta \in \mathscr{H}_{0}\right\}$ is a monotonely increasing net in set of hermitian elements of $\mathscr{Z}$ and therefore the set

$$
\mathscr{H}_{0}\left(A_{0}\right)=\left\{\theta \in \mathscr{H}_{0} \mid \theta(A)=A_{0}=\operatorname{lub}\left\{\theta^{\prime}(A) \mid \theta^{\prime} \in \mathscr{H}_{0}\right\}\right\}
$$

is nonvoid. This is an obvious variation of the statement concerning the set $X$ in Theorem 5 . The set $\mathscr{H}_{0}\left(A_{0}\right)$ is certainly $\mathscr{Z}$-convex and weakly compact. It is a support of $\mathscr{H}_{0}$ for $C \theta(A)+(1-C) \theta^{\prime}(A)=A_{0}$ for $\theta, \theta^{\prime}$ in $\mathscr{H}_{0}$ and $C$ in $\mathscr{Z}$ with $0<C<1$ implies $\theta(A)=\theta^{\prime}(A)=A_{0}$. However the compact support $\mathscr{H}_{0}\left(A_{0}\right)$ of $\mathscr{H}_{0}$ is a compact support of $\mathscr{H}_{1}$ which cannot contain both $\phi$ and $\psi$. This contradicts the maximality of $\mathscr{F}$. Hence we must conclude that $\mathscr{H}_{0}$ contains a single point $\phi_{0}$. We have that $\phi_{0} \neq 0$. On the contrary, if $E$ is an abelian projection in the commutator of $\mathscr{Z}$ which has central support $P$, then $\theta=\operatorname{Re} \tau_{E} \in \mathscr{H}_{1}$ (Corollary, Theorem 5) and $(\theta-\theta) / 2=0$. But $\theta=-\theta=0$ is evidently not true. So $\phi_{0} \neq 0$. Q.E.D.

Let $\mathscr{A}$ be a von Neumann algebra with center $\mathscr{Z}$; a positive functional $\phi \in \mathscr{A}^{\sim}$ is said to be $\mathscr{Z}$-irreducible on $\mathscr{A}$ if given a positive functional $\psi$ in $\mathscr{A} \sim$ with $\psi \leqq \phi$ then there is a $C \in \mathscr{Z}^{+}$such that $C \phi=\psi$.

THEOREM 8. Let $\mathscr{A}$ be a von Neumann algebra with center $\mathscr{Z}$. For a functional $\phi$ 
in the unit sphere $\mathscr{S}$ of the set of positive functionals of $\mathscr{A} \sim$ the following are equivalent: (1) $\phi$ is an extreme point of $\mathscr{S}$; and (2) $\phi(1)$ is a projection of $\mathscr{Z}$ and $\phi$ is $\mathscr{Z}$ irreducible.

Proof. Let $\phi$ be an extreme point of $\mathscr{S}$. Let $P$ be the support of the positive element $\phi(1)$ in the unit sphere of $\mathscr{Z}$. If there is a $\zeta$ in the spectrum $Z$ of $\mathscr{Z}$ such that $0<\phi(1)^{\wedge}(\zeta)<1$, there is an $\varepsilon>0$ and a projection $Q$ majorized by $P$ with $Q^{\wedge}(\zeta)=1$ and $\varepsilon Q \leqq Q \phi(1) \leqq(1-\varepsilon) Q$. So $\|Q \phi\| \leqq 1-\varepsilon$ and $\theta=(1-\varepsilon)^{-1}$. $Q \phi+(1-Q) \phi$. is a point of $\mathscr{S}$. However $\phi=(1-\varepsilon) \theta+\varepsilon(1-Q) \phi$. This gives a contradiction. So we conclude that $\phi(1)=P$. Now let $\psi \in \mathscr{S}$ and $\psi \leqq \phi$. Setting $\theta=\phi-\psi$ we assume that $\theta(1)^{\wedge}(\zeta) \neq 0$ and $\theta(1)^{\wedge}(\zeta) \neq 1$ for some $\zeta \in Z$. There is an $\varepsilon>0$ and a central projection $Q$ such that $Q^{\wedge}(\zeta)=1$ and $\varepsilon Q \leqq Q \theta(1) \leqq(1-\varepsilon) Q$. This means that $B \theta(1)=Q$ and $D \psi(1)=Q$ for suitable $B$ and $D$ in $(\mathscr{Z} Q)^{+}$. Setting $C=((1-Q) / 2)+Q \theta(1)$, we obtain a central element strictly between 0 and 1 satisfying the relation

$$
\phi=C(B \theta+(1-Q) \phi)+(1-C)(D \psi+(1-Q) \phi) .
$$

Because $B \theta+(1-Q) \phi$ and $D \psi+(1-Q) \phi$ are in $\mathscr{S}, D \psi=Q \phi$ and so $Q \psi=Q \psi(1) \phi$. If $\left\{Q_{n}\right\}$ is a maximal set of mutually orthogonal nonzero central projections such that $Q_{n} \psi(1) \phi=Q_{n} \psi$ for each $Q_{n}$, then $\sum Q_{n}=P$. This proves that $\psi=P \psi=\psi(1) \phi$. Thus, we see that $\phi$ is $\mathscr{Z}$-irreducible.

Conversely, let $\phi$ be $\mathscr{Z}$-irreducible and let $\phi(1)$ be a projection $P$. If $\phi=C \psi$ $+(1-C) \theta$ for $\psi, \theta \in \mathscr{S}$ and $0<C<1$ in $\mathscr{Z}$, then there is a $D \in \mathscr{Z}^{+}$such that $C \psi=D \phi$. Then the two relations $C^{-1} D P=\psi(1)$ and $P=C \psi(1)+(1-C) \theta(1)$ imply $P=\psi(1)$. So $\psi=C^{-1} D \phi=\phi$. Similarly $\theta=\phi$. This proves $\phi$ is an extreme point of $\mathscr{S}$. Q.E.D.

THEOREM 9. Let $\mathscr{Z}$ be a commutative von Neumann algebra and let $\mathscr{A}$ be a $C^{*}$ algebra with center $\mathscr{Z}$. Then $\mathscr{A}$ is a type I von Neumann algebra if and only if the module $\mathscr{A}$ is the second dual $\mathscr{M} \sim$ of a Banach $\mathscr{Z}$-module $\mathscr{M}$.

Proof. If $\mathscr{A}$ is a type I von Neumann algebra, then $\mathscr{A}$ is the second dual of the closed two-sided $I_{a}$ of $\mathscr{A}$ generated by the abelian projections of $\mathscr{A}[9, \S 4]$.

Conversely, it is already known that $\mathscr{A}$ is a von Neumann algebra (Theorem 4) and so it is sufficient to prove that every nonzero projection $P$ of $\mathscr{Z}$ majorizes a nonzero abelian projection. Let $\phi$ be an extreme point of the set of positive functionals $\mathscr{S}$ of the unit sphere of $\mathscr{A}_{\sim}$ such that $P \phi \neq 0$. Then it is easy to see that $P \phi$ is an extreme point of $\mathscr{S}$ and so we assume that $P \phi=\phi$. Let $x$ be a unit vector in the Hilbert space $H$ of $\mathscr{A}$ such that $\phi(1) x=x$ (Theorem 8). Let $g$ be a positive functional on $\mathscr{A}$ majorized by $w_{x} \cdot \phi$. If $A \in \mathscr{Z}^{+}$, then $g(A) \leqq w_{x}(\phi(1) A)=w_{x}(A)$. There is an element $C$ in $\mathscr{Z}^{+}$such that $g(A)=(A C x, C x)$ for all $A$ in $\mathscr{Z}$. By Theorem 1 there is a normal functional $\psi$ of the module $\mathscr{A}$ such that $g=w_{C x} \cdot \psi$ because $g$ is normal. If $E$ is the projection of $H$ onto closure $\{A x \mid A \in \mathscr{Z}\}$ then $C^{2} \psi(A) E \leqq \phi(A) E$ for every $A$ in $\mathscr{A}^{+}$since $\left(C^{2} \psi(A) E y, E y\right) \leqq(\phi(A) E y, E y)$ for every $y$ in $E(H)$. However 
the projection $E$ is in the commutator of $\mathscr{Z}$ on $H$ and so if $Q$ is the central support of $E$ we have that $C^{2} Q \psi(A) \leqq Q \phi(A) \leqq \phi(A)$ for every $A$ in $\mathscr{A}^{+}$. There is a $D \in \mathscr{Z}^{+}$ such that $C^{2} Q \psi=D \phi$ (Theorem 8 ). Thus $g(A)=w_{x} \cdot \phi(D A)$ for all $A$ in $\mathscr{A}$. This means that the projection in $\mathscr{A}$ corresponding to closure $\left\{A^{\prime} x \mid A^{\prime} \in \mathscr{A}^{\prime}\right\}$ where $\mathscr{A}^{\prime}$ is the commutator of $\mathscr{A}$ is abelian in $\mathscr{A}[8, \S 2]$. This completes the proof. Q.E.D.

REMARK. Actually we have proved that a normal functional $f$ on $\mathscr{A}$ is centrally reducible (cf. $[8, \S 2])$ if and only if there is a $\mathscr{Z}$-irreducible normal functional $\phi$ of the module $\mathscr{A}$ such that $f \cdot \phi=f$.

\section{BIBLIOGRAPHY}

1. J. Dixmier, Les fonctionelles linéaires sur l'ensemble des opérateurs d'un espace de Hilbert, Ann. of Math. 51 (1950), 387-408.

2. - Les algèbres d'opérateurs dans l'espace hilbertien, Gauthier-Villars, Paris, 1957.

3. - Les $C^{*}$-algèbres et leur représentations, Gauthier-Villars, Paris, 1964.

4. J. Glimm, A Stone-Weierstrass theorem for $C^{*}$-algebras, Ann. of Math. (2) 72 (1960), 216-244.

5. - Type I $C^{*}$-algebras, Ann. of Math. (2) 73 (1961), 572-612.

6. R. Godement, Sur la théorie des représentations unitaires, Ann. of Math. (2) 53 (1951), 68-124.

7. A. Grothendieck, Un résultat sur le dual d'une $C^{*}$-algèbre, J. Math. Pures Appl. (9) 36 (1957), 97-108.

8. H. Halpern, An integral representation of a normal functional on a von Neumann algebra, Trans. Amer. Math. Soc. 125 (1966), 32-46.

9. - A spectral decomposition for self-adjoint elements in the maximum GCR ideal of a von Neumann algebra with applications to noncommutative integration theory, Trans. Amer. Math. Soc. 133 (1968), 281-306.

10. I. Kaplansky, Algebras of type I, Ann. of Math. (2) 56 (1952), 460-472.

11. - Modules over operator algebras, Amer. J. Math. 75 (1953), 839-858.

12. J. Kelly and I. Namioka, Linear topological spaces, Van Nostrand, Princeton, N. J., 1963.

13. S. Sakai, A characterization of $W^{*}$-algebras, Pacific J. Math. 6 (1956), 763-773.

14. - On linear functionals of $W^{*}$-algebras, Proc. Japan Acad. 34 (1958), 571-574.

15. —_, A Radon-Nikodym theorem for $W^{*}$-algebras, Bull. Amer. Math. Soc. 71 (1965), 149-151.

16. R. Schatten, $A$ theory of cross spaces, Princeton Univ. Press, Princeton, N. J., 1950.

17. I. E. Segal, Decomposition of operator algebras. I, II, Mem. Amer. Math. Soc. No. 9 (1951), $67 \mathrm{pp}$. and $66 \mathrm{pp}$.

18. J. Taylor, The Tomita decomposition of rings of operators, Trans. Amer. Math. Soc. 113 (1964), 30-39.

19. Z. Takeda, On the representation of operator algebras. II, Tôhoku Math. J. 6 (1954), 299-304.

20. M. Tomita, Representations of operator algebras, Math. J. Okayama Univ. 3 (1954), 142-173.

IlLinOIS InSTITUTE OF TeChNology, Chicago, Illinois 